\title{
AUTOMORPHISMS OF A CLASS OF METABELIAN GROUPS
}

\author{
BY \\ S. BACHMUTH
}

1. Introduction. The purpose of this paper is to extend the results of [2] and [3] to the groups $U=F / F^{\prime \prime}\left(F^{\prime}\right)^{m}$, where $m$ is a positive integer greater than one. Here $\left(F^{\prime}\right)^{m}$ is the group generated by all $m$ th powers of the commutator subgroup of the finitely generated free group $F$. The notation we use throughout is consistent with that of [2] and [3], but for the convenience of the reader we first recall some definitions and notation which are not standard.

Let $s_{1}, \ldots, s_{q}$ and $a_{1}, \ldots, a_{q}$ denote the generators of $F / F^{\prime} \cong U / U^{\prime}$ and $U$, respectively, which correspond to the free generators $\bar{a}_{1}, \ldots, \bar{a}_{q}$ of the free group $F$ of rank $q$. $U^{\prime}$ becomes a (multiplicative) module over $Z_{m}\left(F / F^{\prime}\right)=\mathscr{R}$, the group ring of $F / F^{\prime}$ over the integers modulo $m$, under the definition

and

$$
\left[a_{i}, a_{j}\right]^{s_{k}}=\left[a_{i}, a_{j}\right]^{a_{k}}=a_{k}\left[a_{i}, a_{j}\right] a_{k}^{-1}
$$

$$
\left[a_{i}, a_{j}\right]^{P+Q}=\left[a_{i}, a_{j}\right]^{P}\left[a_{i}, a_{j}\right]^{Q}
$$

for elements $P, Q$ in $\mathscr{R}$. Here we put $\left[a_{i}, a_{j}\right]=a_{i} a_{j} a_{i}^{-1} a_{j}^{-1}$ and when we write $\left[a_{i}, a_{j}\right]^{P}$ for $P$ in $\mathscr{R}, P$ will be considered as a "polynomial" in the $s_{i}^{ \pm 1}$ or the $a_{i}^{ \pm 1}$, whichever is most convenient at the time.

In this paper we are concerned with the group of IA-automorphisms of $U$, i.e., those automorphisms which induce the identity automorphism on the abelianized group $U / U^{\prime}$. In Proposition 1 , we modify the Magnus representation of $F / F^{\prime \prime}$ (see $\S 3$ of [2]) to get a faithful representation of $U$. Using this proposition, it follows that the representation of the IA-automorphism group of $F / F^{\prime \prime}$ due to W. Magnus (see $\$ 3$ of [2]) becomes a representation of the IA-automorphism group of $F / F^{\prime \prime}\left(F^{\prime}\right)^{m}$ if one restricts the entries to be elements in the ring $\mathscr{R}$.

One can view this as beginning with the Magnus representation of the IAautomorphism group of $F / F^{\prime \prime}$ and factoring out the ideal generated by $m$ from the group ring of the free abelian group over the integers. One may factor out more complicated ideals, and hence, in this paper, we may consider ourselves as working in the simplest possible situation. The results are essentially different depending upon whether or not $m$ is a squarefree integer. In the case where $m$ has a square factor, many of the results of [2] and [3] are no longer valid. We will for the most part restrict ourselves to the case where $m$ is a prime (and give counterexamples for the case of arbitrary $m$ ). This in effect will mean that when $m$ is an integer such

Received by the editors February 14, 1966. 
that $Z_{m}$ is semisimple, the corresponding problems are also solved, for as we shall see, all problems are reducible to the prime spots. For example, although in the semisimple case, Theorem 1 below is no longer valid, it is nevertheless true that all IA-automorphisms are "products" of inner automorphisms corresponding to the primes contained in $m$. These matters will be made precise in a sequel (jointly with $\mathrm{H}$. Mochizuki) where the semisimple as well as the case where $Z_{m}$ contains nilpotent elements will be handled. One of the exceptions in this paper to the restriction that $m$ be a prime is in the determination of the $Z_{m}\left(U / U^{\prime}\right)$ module structure of $U^{\prime}$. We now formulate the main results.

In the case where $m$ is a prime $p$, the analogue of Theorem 1 of [2] carries over without any changes in the proof.

Proposition 2. A necessary and sufficient condition for an $n \times n$ matrix $M(\alpha)$ $=\left(a_{i j}\right)$ over $\mathscr{R}$ to represent an IA-automorphism $\alpha$ of $F / F^{\prime \prime}\left(F^{\prime}\right)^{p}$ is that

(1) det $M(\alpha)=\prod_{i=1}^{q} s_{i}^{j_{1}}$, where $j_{1}, \ldots, j_{q}$ are integers, and

(2) $\sum_{j=1}^{q} a_{i j}\left(1-s_{j}\right)=1-s_{i}$, for $i=1,2, \ldots, q$.

This result is false in the case where $m$ is no longer a prime since $\mathscr{R}$ now has nontrivial units which the $\operatorname{det} M(\alpha)$ may assume.

THEOREM 1. Let $U$ be the group corresponding to the free group of rank 2. If $m$ is a prime, then any IA-automorphism of $U$ is an inner automorphism.

We will give an example later which shows that Theorem 1 is false for general $m$. The proof of Theorem 1 is the same as the corresponding proof for the group $F / F^{\prime \prime}$ given in [2] and hence need not be repeated here.

We emphasize that $m$ is arbitrary in the following theorem.

THEOREM 2. $U^{\prime} \cong F^{\prime} \mid F^{\prime \prime}\left(F^{\prime}\right)^{m}$ as a natural $Z_{m}\left(U / U^{\prime}\right) \cong Z_{m}\left(F / F^{\prime}\right)$ module (written in multiplicative notation) is defined by generators $\left[a_{i}, a_{j}\right]$ for all $i<j$ and relations,

$$
\left[a_{i}, a_{j}\right]^{\left(1-a_{k}\right)}\left[a_{i}, a_{k}\right]^{-\left(1-a_{j}\right)}\left[a_{j}, a_{k}\right]^{\left(1-a_{i}\right)}=1
$$

for all $i<j<k$.

In §3, we will give a proof of Theorem 2 which does not depend upon the proof of Proposition 2 (or of Lemma 1 of [2] upon which the proof of Proposition 2 depends).

We next take up the question of induced automorphisms. The main result here is analogous to the main result for the case of $F / F^{\prime \prime}$ (Lemma 7 or Theorem 2 of [3]) which we formulate as follows: We use the same notation as introduced above and also use $a_{i}$ to mean the cosets $a_{i} U_{n}$, where $U_{n}$ is the $n$th term of the lower central series of $U$. Assume that $\bar{\alpha}$ is an automorphism of $U / U_{n}$ and is described by the mapping

$$
a_{i} \bar{\alpha}=a_{i} \prod_{j<k}\left[a_{j}, a_{k}\right]^{P_{i j k}},
$$

where $P_{i j k}=\sum a_{i j k}(t) t$, the summation being over distinct monomials $t$ in the $\sigma_{i}=\left(1-a_{i}\right)(i=1,2, \ldots, q)$ and the $a_{i j k}(t)$ are integers. 
THEOREM 3. (1) Assume $m$ is a prime, and that the above automorphism $\bar{\alpha}$ induces the identity on $U / U_{n-1}$. Then for $n \geqq 4, \bar{\alpha}$ is induced by an automorphism of $U$ if and only if we have

$$
\sum_{i<k} a_{i i k}\left(t / \sigma_{k}\right)-\sum_{i>j} a_{i j i}\left(t / \sigma_{j}\right) \equiv 0(\bmod p)
$$

for each monomial $t$ of degree $n-2$. (The summations in (*) are over those pairs $i, k$ (resp. $i, j)$ such that $\sigma_{k}$ (resp. $\sigma_{j}$ ) divides $t$.)

(2) If $m$ is arbitrary, then for $n \geqq 4, \bar{\alpha}$ is induced by an automorphism of $U$ whenever (*) is satisfied.

We will give an example later to show that the converse of (2) is false if $m$ is not squarefree.

REMARK. The question of induced automorphisms in the case of general $m$ has in effect been completely solved. The results appear in a joint paper with H. Mochizuki which is a sequel to this paper. In particular, note Corollaries 3.2, 3.3 and Theorem 4.2 of the sequel.

Theorem 3 gives no information in the case of induced automorphisms of $U / U_{3} \cong F / F_{3}\left(F^{\prime}\right)^{p}$. But here, a direct calculation as done in [1] for the case $F / F_{3}$ will show that all automorphisms of $U / U_{3}$ are induced by automorphisms of $U$ (in fact by automorphisms of $F$ ). Finally, we have as before, the interesting

THEOREM 4. Suppose $m$ is a prime. If an automorphism of $U / U_{4}$ can be induced by an automorphism of $U$, it can be induced by an automorphism of $F$.

I would like to thank the referee for his helpful comments and suggestions, and in particular, for suggesting the very simple proof of Proposition 1.

2. The representation of the IA-automorphism group of $U$. We denote the group ring of a group $G$ over a ring $R$ by $R(G)$. The following is a special case of a much more general theorem due to Magnus [5].

Suppose $\Phi=F / F^{\prime \prime}$ is freely generated by $b_{1}, \ldots, b_{q}$ corresponding to the free generators $\bar{a}_{1}, \ldots, \bar{a}_{q}$, of $F$. Then the mapping of $\Phi$ into the group of matrices

$$
b_{i} \rightarrow\left(\begin{array}{cc}
s_{i} & t_{i} \\
0 & 1
\end{array}\right) \quad(i=1, \ldots, q)
$$

is an isomorphism, where $s_{i}$ is a generator of $F / F^{\prime}$ corresponding to the generator $\bar{a}_{i}$ under the natural map and $t_{1}, \ldots, t_{q}$ are a basis for a free $Z\left(F / F^{\prime}\right)$-module. We state the following lemma from an earlier paper (Lemma 1 of [2]).

LEMMA 1. Let $S=s_{1}^{j_{1}} s_{2}^{j_{2}} \cdots s_{q}^{j_{q}}$ where the $j_{i}$ are arbitrary integers. Let $\gamma_{1}, \ldots, \gamma_{n}$ be elements of $Z\left(F / F^{\prime}\right)$. Then the matrix

$$
\left(\begin{array}{cc}
S & \sum \gamma_{i} t_{i} \\
0 & 1
\end{array}\right)
$$


represents an element of $\Phi$ if and only if the $\gamma_{i}$ satisfy the identity

$$
\sum \gamma_{i}\left(1-s_{i}\right)=1-S \text {. }
$$

The following proposition is a special case of a more general result (see [7]), but because of its central importance to this paper we include a proof.

Proposition 1. If we set $m t_{i}=0$ for each $i$ in the above matrix group, the resulting matrix group is isomorphic to $U=F / F^{\prime \prime}\left(F^{\prime}\right)^{m}$.

Proof. It is clear that we get at least a homomorphic image of $U$. We show that it is in fact an isomorphism. Suppose $x$ in the Magnus representation of $\Phi$ is in the kernel of our homomorphism. Then obviously $x$ is in $\Phi^{\prime}$ and hence $x$ is given by

$$
x=\left(\begin{array}{cc}
1 & \sum m \gamma_{i} t_{i} \\
0 & 1
\end{array}\right)
$$

for some $\gamma_{i}$ in $Z\left(F / F^{\prime}\right)$. Now by the above lemma, we have $m \sum \gamma_{i}\left(1-s_{i}\right)=0$, which implies $\sum \gamma_{i}\left(1-s_{i}\right)=0$. Hence

$$
y=\left(\begin{array}{cc}
1 & \sum \gamma_{i} t_{i} \\
0 & 1
\end{array}\right)
$$

is in the Magnus representation of $\Phi$ and $y^{m}=x$. Thus $x$ is an $m$ th power of an element of $\Phi^{\prime}$, and this completes the proof of Proposition 1.

REMARK. In proving Proposition 1, one may avoid the use of Lemma 1 of [2] by utilizing a free basis for $\Phi^{\prime}$ (e.g., the basis given by Gruenberg [4], Theorem 5.1) and analyzing what the powers of these basis elements look like in the Magnus representation.

We now proceed exactly as in [2] and [3], to define the Magnus representation of the IA-automorphism group of $U$. For convenience we repeat the definition.

If $\alpha$ is an IA-automorphism of $U$ and

$$
a_{i} \alpha=a_{i} \prod_{j<k}\left[a_{j}, a_{k}\right]^{A_{i j k}}
$$

where the $A_{i j k}$ are elements of $\mathscr{R}$, then

$$
M(\alpha)=I+N(\alpha)
$$

where $I$ is the identity matrix and $N(\alpha)=\left(d_{u v}\right)$ is given by

$$
d_{u v}=s_{u}\left[\sum_{h>v}\left(1-s_{h}\right) A_{u v h}-\sum_{h<v}\left(1-s_{h}\right) A_{u h v}\right] .
$$

It is no longer true that $\mathscr{R}=Z_{m}\left(F / F^{\prime}\right)$ has only trivial units. We have, however, trivially,

LeMma 2. If $p$ is a prime, then $\mathscr{R}=Z_{p}\left(F / F^{\prime}\right)$ has only trivial units. 
COROLlARY. If $m$ is a prime and $\alpha$ is an IA-automorphism of $U$, then

$$
\operatorname{det} M(\alpha)=\prod_{i=1}^{q} s_{i}^{j_{i}} .
$$

Proof. By Lemma 1 , det $M(\alpha)=a \prod_{i=1}^{q} s_{i}^{j_{1}}$ for some $a$ in $Z_{p}$. But setting $s_{i}=1$ for each $i$ (i.e., factoring by the augmentation ideal) turns $M(\alpha)$ into the identity matrix by (2.1) and (2.2) and hence it follows that $a=1$.

REMARK. If $m$ is not a prime, then Lemma 2 is of course no longer true. But more important the corollary is also false, and it is this fact which invalidates most of the results for the case where $m$ is no longer squarefree. (In the case where $Z_{m}$ contains no nilpotent elements, the corollary becomes det $M(\alpha)=\sum_{k} \prod_{i} u_{k} s_{i}^{s^{i}}$, where $1=\sum u_{k}$ is the unique decomposition of 1 in $Z_{m}$ into orthogonal idempotents. However, for arbitrary $m$, the situation is more serious.) Translated in practical language, this means there exist "more" automorphisms of $F / F^{\prime \prime}\left(F^{\prime}\right)^{m}$ than in the case where $m$ is squarefree or $m$ is zero. We given an illustration before proceeding.

EXAMPLE. Let $m=4$ and let $\alpha$ be the endomorphism of $U$ for which $a_{1} \alpha$ $=a_{1}\left[a_{1}, a_{2}\right]^{2 a_{1}^{-1}}, a_{2} \alpha=a_{2}$. Then $\alpha^{2}=1$ and hence $\alpha$ is an automorphism. This is most easily seen by going to the Magnus representation. We have

$$
M(\alpha)=\left(\begin{array}{cc}
3-2 s_{2} & 0 \\
-2\left(1-s_{2}\right) & 1
\end{array}\right),
$$

and a direct calculation shows that $M^{2}(\alpha)=I$. Notice that $\operatorname{det} M(\alpha)=3-2 s_{2}$ which is a nontrivial unit of $\mathscr{R}$; i.e., $\left(3-2 s_{2}\right)^{2}=1$. This example also shows that Theorem 1 is false for $m=4$. In fact the above automorphism is in no way decomposable as a product of inner automorphisms of appropriate quotient groups (as will be seen to be the case for $m$ squarefree).

3. Proof of Theorem 2. We begin by giving some remarks necessary for the proof. Proposition 2 of $\S 1$ enables us to construct IA-automorphisms of $U$. In particular, any mapping $\alpha$ of $U$ of the form

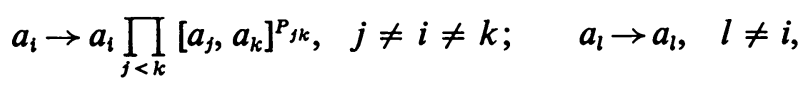

where the $P_{i j}$ are any elements of $\mathscr{R}$, is always an IA-automorphism of $U$ which has determinant equal to one. We will call these automorphisms "exceptional automorphisms."

In the case where $U=F / F^{\prime \prime}(F)^{m}$ has rank 2, the Jacobi product (1.1) is vacuous, and hence Theorem 2 below says that $F^{\prime} \mid F^{\prime \prime}\left(F^{\prime}\right)^{m}$ is a free $\mathscr{R}$-module. We remind the reader that $m$ is now assumed arbitrary.

THeOREM 2. Let $a_{1}, a_{2}, \ldots, a_{q}$ be free generators of $U$, and $P_{i j}$ elements of $\mathscr{R}$. Then the only nontrivial relations involving commutators of the form $\left[a_{i}, a_{j}\right]^{P_{i s}}$ are products of transforms of the Jacobi identity (1.1). 
Proof. Suppose we have a nontrivial relation

$$
\prod_{i<j}\left[a_{i}, a_{j}\right]^{P_{1 j}}=1 \text {. }
$$

We wish to show that the left-hand side is a product of transforms of Jacobi products. The idea involved is to use the existence of the automorphisms termed exceptional above to derive relations on the $P_{i j}$ and then to deduce that these relations are just the Jacobi relations. We rewrite (3.1) as

$$
\prod_{1<k}\left[a_{1}, a_{k}\right]^{P_{1 k}}=\prod_{i<j: i \neq 1}\left[a_{i}, a_{j}\right]^{-P_{i j}}
$$

and consider the exceptional automorphism $\alpha$ defined by

$$
a_{1} \alpha=a_{1} \prod_{i<j: i \neq 1}\left[a_{i}, a_{j}\right]^{-P_{i j},} \quad a_{i} \alpha=a_{i}, \quad i \neq 1 .
$$

As noted above, $\alpha$ has determinant one. But because of (3.2), this automorphism is the same as

$$
a_{1} \alpha=a_{1} \prod_{k=2}^{q}\left[a_{1}, a_{k}\right]^{P_{1 k}}, \quad a_{i} \alpha=a_{i}, \quad i \neq 1
$$

which has determinant equal to

We therefore have

$$
1+\sum_{j=2}^{q} s_{1}\left(1-s_{j}\right) P_{1 j}
$$

$$
\sum_{j=2}^{q}\left(1-s_{j}\right) P_{1 j}=0 \text {. }
$$

It is easy to verify that all solutions of (3.3) are sums of solutions of the form

$$
\begin{aligned}
& P_{1 u}=\left(1-s_{v}\right) X, \quad u \neq v, u \neq 1, v \neq 1 \\
& P_{1 v}=-\left(1-s_{u}\right) X, \\
& P_{1 l}=0, \quad l \neq u, l \neq v, l \neq 1,
\end{aligned}
$$

where $X$ is an arbitrary element of $\mathscr{R}$.

Let us consider one of the cases of (3.4), i.e., fix $u$ and $v$ and let $X$ be any element of $\mathscr{R}$. Without loss of generality we may take $u=2, v=3$. With this as the solution of (3.3), we may use the Jacobi identity to replace $\left[a_{1}, a_{2}\right]^{P_{12}}\left[a_{1}, a_{3}\right]^{P_{13}}$ by $\left[a_{2}, a_{3}\right]^{-\left(1-a_{1}\right) X}$ in equation (3.2). Equation (3.2) now becomes

$$
\left[a_{2}, a_{3}\right]^{P_{23}-\left(1-a_{1}\right) x}=\prod_{i<j ; i \neq 1 ;(i, j) \neq(2,3)}\left[a_{i}, a_{j}\right]^{-P_{i j}}
$$

Notice that if $q=3$, we would be finished since the solution of (3.3) chosen is the general solution and the right-hand side of (3.5) is vacuously the identity element of $U$ and we would therefore have $P_{23}=\left(1-a_{1}\right) X$. Suppose therefore $q>3$ and we assume the theorem is true for the case $q-1$. We consider the mapping $\beta$ defined by

$$
a_{q} \beta=a_{q}\left[a_{2}, a_{3}\right]^{P_{23}-\left(1-a_{1}\right) x}, \quad a_{i} \beta=a_{i}, \quad i \neq q .
$$


Since $q>3, \beta$ is an automorphism of $U$ and $\beta$ has determinant one. But, by (3.5) $\beta$ is the automorphism

$$
\begin{aligned}
& a_{q} \beta=a_{q} \prod_{i<j: i \neq 1:(i, j) \neq(2,3)}\left[a_{i}, a_{j}\right]^{-P_{i j}} \\
& a_{i} \beta=a_{i}, \quad i \neq q
\end{aligned}
$$

which has determinant equal to $1+\sum_{j=2}^{q-1} s_{q}\left(1-s_{j}\right) P_{j q}$. Hence we have

$$
\sum_{j=2}^{q-1}\left(1-s_{j}\right) P_{j q}=0 \text {. }
$$

The solutions of (3.6) are sums of solutions of the form

$$
\begin{aligned}
& P_{u q}=\left(1-s_{v}\right) Y_{u v}, \\
& P_{v q}=-\left(1-s_{u}\right) Y_{u v}, \\
& P_{k q}=0, \quad k \neq u, k \neq v,
\end{aligned}
$$

where $u, v$ may take on values from among $2,3, \ldots, q-1$ and $Y_{u v}$ is any element of $Z_{m}\left(F / F^{\prime}\right)$. Now suppose for a particular $u, v, Y_{u v}$ is nonzero. Then we may use the Jacobi identity to replace $\left[a_{u}, a_{q}\right]^{P_{u q}}\left[a_{v}, a_{q}\right]^{P_{v q}}$ by $\left[a_{u}, a_{v}\right]^{Q_{u v}}$, where $Q_{u v}$ $=\left(1-s_{q}\right) Y_{u v}$. In this manner we remove all the elements on the left-hand side of (3.1) of the form $\left[a_{i}, a_{q}\right]^{P_{i q}}$. There remain only commutators of the form $\left[a_{j}, a_{k}\right]^{P_{j k}}$ where $k<q$, and we may now apply our induction hypothesis. We deal with other particular solutions of (3.3) in a similar manner. The general solution of (3.3) is a sum of the particular solutions. Each such solution leads to Jacobi products and the sum of these solutions leads to the product of these various Jacobi products. This completes the proof of Theorem 3 .

4. Proof of Theorems 3 and 4. Our presentation depends upon the following fundamental result due to W. Magnus. A proof may be found in [6, Chapter IV].

PROPOSITION 3. Let $\mathscr{M}$ be the variety of all metabelian nilpotent groups of class $c$. Then any element of any group $G$ in $\mathscr{M}$ can be represented as a product of (left normed) basic commutators in $G$.

In particular, this result applies to the groups $U / U_{n}$. Thus, we can state the following fundamental lemma, whose proof now goes over word for word the same as the proof of Lemma 4 in [3]. We use the same notation as in [3] and perhaps for convenience, we list the definitions here.

$\Sigma$ is the augmentation ideal of $\mathscr{R}$ generated by all elements $\sigma_{i}=\left(1-s_{i}\right)$, for $i=1, \ldots, q . \Sigma^{k}$ is the $k$ th power of $\Sigma . A\left(U ; U / U_{n}\right)$ is the group of automorphisms of $U$ which induces the identity automorphism on $U / U_{n} . A^{*}\left(U / U_{n} ; U / U_{n-1}\right)$ is the subgroup of $A\left(U / U_{n} ; U / U_{n-1}\right)$ consisting of those automorphisms which are induced by automorphisms of $U$. The elements $a_{1}, \ldots, a_{q}$ will be used to denote free generators of $U$ and also the corresponding free generators of $U / U_{n}$.

LeMma 3. If $\alpha$ is in $A\left(U ; U / U_{n}\right)$, then $M(\alpha) \equiv I$ (modulo $\left.\Sigma^{n-1}\right)$. 
Now the question of which automorphisms of $U / U_{n}$ are induced by automorphisms of $U$, reduces to the same question for $A=A\left(U / U_{n} ; U / U_{n-1}\right)$. We therefore wish to determine the subgroup $A^{*}=A^{*}\left(U / U_{n} ; U / U_{n-1}\right)$ of $A$. We proceed exactly as in $\S 5$ of [3]. For $\beta$ in $A$ we write

$$
a_{i} \beta=a_{i} \prod_{j<k}\left[a_{j}, a_{k}\right]^{A_{i j k}}
$$

where $A_{i j k}$ are polynomials all of whose terms are of degree $n-3$ in the $\sigma_{i}=1-a_{i}$ over $Z_{m}$. We define $N_{1}(\beta)$ exactly as in [3]; i.e., we formally write $M(\beta)=I+N(\beta)$ using (2.1) and (2.2) and then $N_{1}(\beta)$ is the image of $N(\beta)$ in $\Sigma^{n-2} / \Sigma^{n-1}$. We now restrict ourselves to the case where $m$ is a prime. Our aim is to show that $\beta$ is in $A^{*}$ if and only if trace $N_{1}(\beta)=0$. The following is the necessary modification of Lemma 6 of [3].

LEMMA 4. If $\alpha$ is an automorphism of $U$ and $n \geqq 4$, then, $\alpha$ induces $\beta$ in $A^{*}\left(U / U_{n} ; U / U_{n-1}\right)$ only if $\operatorname{det} M(\alpha)=\prod_{i=1}^{q} s_{i}^{j_{i}}$, where the $j_{i}$ are multiples of $p$. (The converse is obvious, i.e., if $\operatorname{det} M(\beta)=\prod s_{i}^{j_{1}}$, then by Proposition $2, \beta$ as given by 4.1 may be thought of as an automorphism of $U$ rather than $U / U_{n}$.)

Proof. By Lemma 2, det $M(\alpha)=\prod_{i=1}^{q} s_{i}^{j_{1}}$ for integers $j_{1}, \ldots, j_{q}$. We have, by Lemma 3 , that $M(\alpha) \equiv I\left(\bmod \Sigma^{n-2}\right)$ and hence $\operatorname{det} M(\alpha) \equiv 1\left(\bmod \Sigma^{n-2}\right)$. But $\operatorname{det} M(\alpha)=\prod_{i=1}^{q}\left(1-\sigma_{i}\right)^{j_{i}}$ and we must therefore have

$$
\left(\begin{array}{c}
j_{i} \\
1
\end{array}\right) \equiv 0 \quad(\bmod p) \text { for }(i=1,2, \ldots, q)
$$

or else det $M(\alpha)$ contains terms of degree smaller than $n-2$ in the $\sigma_{i}$.

REMARK. If $m$ is arbitrary, then Lemma 4 is not true because we cannot assert that det $M(\alpha)=\prod_{i=1}^{q} s_{i}^{j_{i}}$. However, if $m$ is squarefree, then det $M(\alpha)$ is indeed of the form $\prod_{i=1}^{q} s_{i}^{j}$ at each prime spot. This will enable us to assert in the case of squarefree $m$, that if trace $N_{1}(\beta) \equiv 0(\bmod m)$, then $\beta$ is induced by an automorphism of $U$, and conversely. For arbitrary $m$, the converse if no longer valid because $U$ now has too many automorphisms. We will give an example later of an element $\beta$ in $A\left(U / U_{4} ; U / U_{3}\right)$ for which trace $N_{1}(\beta) \not \equiv 0$, but nonetheless $\beta$ is in $A^{*}\left(U / U_{4} ; U / U_{3}\right)$.

For the case where $m$ is a prime, the procedure for showing that $\beta$ as given by (4.1) is in $A^{*}$ if and only if trace $N_{1}(\beta)=0$ proceeds now as in the case $m=0$ developed in $\S 5$ of [3]. We sketch the procedure.

If $\alpha$ is an automorphism of $U$ and induces $\beta$ in $A^{*}\left(U / U_{n} ; U / U_{n-1}\right)$ (where $n \geqq 4$ ), then by Lemma 4 , we have

where

$$
\operatorname{det} M(\alpha)=\prod_{i=1}^{q} s_{i}^{j_{i}}=\prod_{i=1}^{q}\left(1-\sigma_{i}\right)^{j_{i}},
$$

$$
\left(\begin{array}{l}
j_{i} \\
k
\end{array}\right) \equiv 0(\bmod m) \text { for } k=1, \ldots, n-3
$$


But det $M(\alpha)=1+$ trace $N(\alpha)+$ terms quadratic or higher in the $A_{i j k}$. Hence modulo $\Sigma^{n-1}$, this reduces to trace $N(\alpha) \equiv 0$ and hence trace $N_{1}(\alpha)=0$. This gives our result in one direction.

For the converse, we are given that trace $N_{1}(\beta)=0$. We first interpret this condition in an explicit manner as follows. Trace $N_{1}(\beta)=0$ is a linear condition on the coefficients of the polynomials $A_{i j k}$ regarded as polynomials in $\sigma_{i}(i=1,2$, $\ldots, q)$. Thus write $A_{i j k}=\sum a_{i j k}(t) t$ where the summation is over distinct monomials $t$ in the $\sigma_{i}(i=1, \ldots, q)$, and the $a_{i j k}(t)$ are elements of $Z_{p}$. Then trace $N_{1}(\beta)=0$ is equivalent to the following system of linear conditions on the coefficients $a_{i j k}(t)$.

$$
\sum_{i<k} a_{i t k}\left(t / \sigma_{k}\right)-\sum_{i>j} a_{i j i}\left(t / \sigma_{j}\right)=0 .
$$

There is one equation for each $t$ of degree $n-2$ and all these equations are independent. (The summations in (4.2) are over those pairs $i, k$ (or $i, j$ ) such that $\sigma_{k}$ (resp. $\sigma_{j}$ ) divides $t$. The proof of the converse now proceeds exactly as in [3]. That is, for any solution of (4.2) one constructs an automorphism of $U$ having polynomials $A_{i j k}$ for which the terms of degree less than $n-3$ are zero and those of degree $n-3$ the given solution, exactly as in Lemma 7 of [3]. These constructions do not depend upon the fact that $m$ is a prime since $m$ does not enter into these constructions. This remark accounts for part (2) of the following, which is a formal statement of the preceding discussion.

THeORem 3. (1) Let $m$ be a prime. Then for $n \geqq 4$ and $\beta$ in $A\left(U / U_{n} ; U / U_{n-1}\right)$, $\beta$ is in $A^{*}\left(U / U_{n} ; U / U_{n-1}\right)$ if and only if trace $N_{1}(\beta)=0$.

(2) Suppose $m$ is arbitrary. Then for $n \geqq 4$ and $\beta$ in $A\left(U / U_{n} ; U / U_{n-1}\right), \beta$ is in $A^{*}\left(U / U_{n} ; U / U_{n-1}\right)$ if trace $N_{1}(\beta)=0$.

It is not true that $\beta$ in $A^{*}\left(U / U_{n} ; U / U_{n-1}\right)$ implies trace $N_{1}(\beta)=0$, as the following example shows.

EXAMPLE. Take $m=4$ and let $a_{1} \alpha=a_{1}\left[a_{1}, a_{2}\right]^{A}$, where $A=2 a_{1}^{-1}\left(1+a_{2}\right)$ and $a_{2} \alpha=a_{2}$. One easily checks that $\alpha$ is an automorphism of $U$ with $\operatorname{det} M(\alpha)=3-2 s_{2}^{2}$. What automorphism $\beta$ of $U / U_{4}$ does $\alpha$ induce? We have, working in $\mathscr{R}$ and also working modulo $\Sigma^{2}$, that $a_{1}^{-1} \equiv 1+\sigma_{1} \bmod \Sigma^{2}$ and $1+a_{2}=2-\sigma_{2}$ and therefore

$$
2 a_{1}^{-1}\left(1+a_{2}\right) \equiv 2\left(1+\sigma_{1}\right)\left(2-\sigma_{2}\right) \equiv 2 \sigma_{2} \quad\left(\bmod \Sigma^{2}\right) .
$$

Thus $a_{1} \beta=a_{1}\left[a_{1}, a_{2}\right]^{\left(1-a_{2}\right)}, a_{2} \beta=a_{2}$. Hence we have $a_{112}\left(\sigma_{2}\right)=2, a_{112}\left(\sigma_{1}\right)$ $=a_{212}\left(\sigma_{1}\right)=a_{212}\left(\sigma_{2}\right)=0$. But the condition trace $N_{1}(\beta)=0$ (i.e., (4.2)) for the case $q=2$ and $n=4$ is $a_{212}\left(\sigma_{1}\right)=a_{112}\left(\sigma_{2}\right)=0, a_{112}\left(\sigma_{1}\right)-a_{212}\left(\sigma_{2}\right)=0$, which is not satisfied for $\beta$.

Our last result now follows exactly as Theorem 4 of [3]. As is clear, the same automorphisms of the free group $F$ used in the proof of Theorem 4 of [3] may be used in Theorem 4 below. 
THEOREM 4. (1) Let $m$ be a prime. Then any automorphism of $U / U_{4}$ which is induced by an automorphism of $U$, can be induced by an automorphism of $F$.

(2) Let $m$ be arbitrary. Then any automorphism $\beta$ of $U / U_{4}$ which has the property trace $N_{1}(\beta)=0$ can be induced by an automorphism of $F$.

\section{REFERENCES}

1. S. Andreadakis, Automorphisms of free groups and free nilpotent groups, Proc. London Math. Soc. 15 (1965), 239-268.

2. S. Bachmuth, Automorphisms of free metabelian groups, Trans. Amer. Math. Soc. 118 (1965), 93-104.

3. - Induced automorphisms of free groups and free metabelian groups, Trans. Amer. Math. Soc. 122 (1966), 1-17.

4. K. W. Gruenberg, Residual properties of infinite soluble groups, Proc. London Math. Soc. 12 (1957), $29-61$.

5. W. Magnus, On a theorem of Marshall Hall, Ann. of Math. 40 (1939), 764-768.

6. H. Neumann, Varieties of groups, Lectures delivered at the Manchester College of Science and Technology 1962-1963. Lecture notes by I. M. S. Dey and C. H. Houghton.

7. S. Bachmuth and I. Hughes, Centers of certain presentations of finite groups, Abstract 636-4, Notices Amer. Math. Soc. 13 (1966), 576.

\section{University of CaLIForNia,}

Santa Barbara, California 